\title{
Green Synthesis of Selenium Nanoparticle Using Leaves Extract of Withania somnifera and Its Biological Applications and Photocatalytic Activities
}

\author{
Venkatesan Alagesan $^{1} \cdot$ Sujatha Venugopal ${ }^{1}$ \\ Published online: 3 November 2018 \\ (C) The Author(s) 2018
}

\begin{abstract}
The green Se NPs (selenium nanoparticles) are formed by simple mixing of Withania somnifera (W. somnifera) leaves extract and selenious acid $\left(\mathrm{H}_{2} \mathrm{SeO}_{3}\right)$ solution. This mixture was stirred which gave a dispersion of Se NPs conjugated with W. somnifera secondary metabolites. The work was focused to determine the phytochemical analysis of leaves extract, green synthesis, its characterization and its applications. Screening analysis showed enormous phytoconstituents in leaves aqueous extract and simultaneously, the synthesized Se NPs by FT-IR spectrum confirm the presence of functional groups which were associated with bioactive molecules. The total flavonoid, phenolic and tannin contents in the aqueous extract were found to be 12.74, 40.54, and $156.33 \mu \mathrm{g} / \mathrm{mg}$ respectively. The suspension solution confirms the formation of Se NPs showed ( $310 \mathrm{~nm})$ by UV analysis. Xray diffraction study exhibits the amorphous nature of Se NPs. Se NPs were spherical in shape within the diameter range of 45$90 \mathrm{~nm}$ by FE-SEM. The intense, narrow width of selenium has high purity and was identified using EDX. The TEM analysis exhibited the amorphous nature of particles. Green synthesized Se NPs were found to possess significant antioxidant activity $\left(\mathrm{IC}_{50}-14.81 \mu \mathrm{g} / \mathrm{mg}\right)$ and considerable antibacterial activity on Bacillus subtilis $(12 \mathrm{~mm})$, Klebsiella pneumoniae $(14 \mathrm{~mm})$ and Staphylococcus aureus $(19.66 \mathrm{~mm})$. Antiproliferative effects of Se NPs possess great growth control against A549 cells $\left(\mathrm{IC}_{50}\right.$ at $25 \mu \mathrm{g} / \mathrm{ml})$. Se NPs exhibit efficient methylene blue (MB) dye degradation in the presence of sunlight. The present results support the advantages of green method for the production of Se NPs having potential activities.
\end{abstract}

\section{Highlights}

-Withania somnifera leaves extract possesses active phytoconstituents and acts as reducing agents for the preparation of Se NPs and the capping by the phytoconstituents providing stability to Se NPs as evident from FT-IR studies.

- The green synthesized metal nanoparticle has emerged at low cost, simpler and better choice than physical and chemical methods.

- Green synthesized Se NPs were found to be almost spherical in shape with particle size around 40-90 nm, selenium has high purity and crystalline nature was identified using EDX and XRD.

- The DPPH scavenging analysis of the amorphous Se NPs has significant antioxidant activity and serves as a potential antibacterial agent to treat diseases caused by bacteria.

- Antiproliferative activity results suggest that Se NPs possess higher growth control against A549 lung carcinoma cancer cells $\left(\mathrm{IC}_{50}\right.$ at $\left.25 \mu \mathrm{g} / \mathrm{ml}\right)$ which indicated their potential in medical applications.

- The photocatalytic study concludes that these Se NPs have efficiency to degrade MB under sunlight irradiation. Therefore, they can find application in water treatment plants and textile industries.

Keywords Withania somnifera $\cdot$ Selenium NPs $\cdot$ Antioxidant $\cdot$ Antibacterial $\cdot$ Photocatalytic activity

Sujatha Venugopal

chemsujatha888@gmail.com

1 Phytochemistry Lab, Department of Chemistry, Periyar University, Periyar Palkalai Nagar, Salem, Tamil Nadu 636011, India

\section{Introduction}

Plants have been explored successfully for rapid biosynthesis of metal nanoparticles such as gold [1], silver [2], selenium [3], $\mathrm{MgO}[4], \mathrm{CuO}[5]$ and $\mathrm{ZnO}$ [6] nanoparticles. The 
nanoparticles are used extensively in cancer drug delivery as the drugs which are bound with nanoparticles are able to penetrate deep into the organs [7]. Particularly, essential dietary micronutrient, selenium found in the form of Se NPs, is relatively a new member of drug nanocarriers in medicine [8] because Se NPs exhibited strong antioxidative [9] and antibacterial activity [10].

In recent times, green synthesis using medicinal plant extract has obtained specific importance in the formation of nanoparticles. Especially, phytochemicals are backbone of plants and easily produce nanoparticles with less toxicity. Withania somnifera Dunal (Ashwagandha) - Indian ginseng or winter cherry-is a significant herb in the Ayurvedic medical systems and proved itself as a multipurpose agent [11]. W. somnifera contains active ingredients of withanolide and flavonoids. W. somnifera has antioxidant, anti-inflammatory, anti-tumor, anti-stress, mind-boosting and rejuvenating properties [12]. $W$. somnifera, used as an antioxidant, eliminates free radicals from the human immune system [13]. According to Indian Herbal System (Ayurveda), W. somnifera is considered as an important herb and the best adaptogen [11] and commonly used in a wide range of health benefits. Hence, the current study was intended to use W. somnifera as a bio-reductant and capping agent for the synthesis of Se NPs as a hopeful carrier and to evaluate its antioxidant, antibacterial and photocatalytic activities.

\section{Materials and Methods}

\subsection{Plant Collection and Materials}

Healthy $W$. somnifera leaves were collected from Sarabanga River, Omalur, Salem District, Tamil Nadu, India. W. somnifera was identified and confirmed by the Botanical Survey of India (BSI/SRC/5/23/2014-15/Tech.170), Coimbatore, Tamil Nadu, India.

Selenious acid $\left(\mathrm{H}_{2} \mathrm{SeO}_{3}\right)$ and ethanol were purchased from Merck. All the experiments were performed with sterile distilled water and de-ionized water.

\subsection{Preparation of Plant Leaves Extract}

W. somnifera leaves were washed with sterile distilled water to remove dust particles and then shade dried. W. somnifera leaves extract was prepared by placing $10 \mathrm{~g}$ of dried fine cut in a $500-\mathrm{ml}$ beaker along with $500 \mathrm{ml}$ of de-ionized water. The mixture was boiled for 15 min until the colorless aqueous solution changed to yellow. The mixture was subjected to cooling at room temperature and filtered with (Whatman no.

1) filter paper before centrifuging at $1500 \mathrm{rpm}$ for $5 \mathrm{~min}$ to remove biomaterials. The extract was stored at $25{ }^{\circ} \mathrm{C}$ in order to be used for further experiments.

\subsection{Phytochemical Analysis}

\subsubsection{Qualitative Phytochemical Analysis}

The preliminary phytochemical screening analysis was carried out in aqueous extract of W. somnifera [14].

\subsubsection{Quantitative Phytochemical Analysis}

Determination of Total Phenol Content The total phenol content was estimated by a spectrophotometric assay in aqueous extract of $W$. somnifera [15]. The results were expressed as microgram of gallic acid equivalents (GAEs) per milligram of extract.

Determination of Total Flavonoid Content The total flavonoid content was determined by a spectrophotometric method in the aqueous extract of $W$. somnifera [16]. The results were expressed as microgram of $( \pm$ ) catechin equivalents (CEs) per milligram of extract.

Estimation of Tannin Content Tannin content was measured by Folin-Denis method in the aqueous extract of W. somnifera [17]. The results were expressed as microgram of tannic acid equivalents per milligram of extract.

\subsection{Synthesis of Se NPs}

Green synthesis of Se NPs One hundred milliliters of plant extract was mixed with $50 \mathrm{mM}$ selenious acid and stirred. The solution was incubated at room temperature. The reaction mixture was observed for any change in color and analyzed time to time by a UV-Vis spectrophotometer. After completion of the reaction, the synthesized Se NPs were harvested by centrifuging the reaction mixture at $1500 \mathrm{rpm}$. The nanopellets were washed with distilled water and acetone, and the nanopellet was dried overnight. The red Se NPs were suspended in PBS (pH 7.4) by ultrasonication and then centrifuged. The red Se NPs were used for further analysis.

\subsection{Characterization Techniques}

\subsubsection{UV-Visible Spectroscopy}

The Se NPs were characterized in a PerkinElmer UVVis spectrophotometer, Lambda-19 to know the kinetic behavior of Se NPs. The scanning range of the samples was $200-800 \mathrm{~nm}$ at a scan speed of $480 \mathrm{~nm} / \mathrm{min}$. The data in the spectrophotometer were recorded and 
analyzed by "UV Winlab" software. The UV-Vis absorption spectra of the Se NPs were recorded and "Origin 8" was used to plot the numerical data.

\subsubsection{Fourier Transform Infrared Spectroscopy}

Two milligrams of the nanoparticles was encapsulated in $100 \mathrm{mg}$ of $\mathrm{KBr}$ pellet in order to prepare translucent sample discs. The pelleted sample specimens were subjected to Fourier transform infrared (FT-IR) spectroscopy (Bruker IR Affinity, Japan) in the range of wavelength 400 to $4000 \mathrm{~cm}^{-1}$ with a resolution of $1 \mathrm{~cm}^{-1}$. A PerkinElmer spectrophotometer was employed to record FT-IR spectrum.

\subsubsection{XRD}

The crystalline size and purity were characterized by an $\mathrm{X}$-ray diffractometer (Philips PANanalytical) using $\mathrm{Cu}$ $\mathrm{K} \alpha$ radiation of wavelength $\lambda=1.541 \AA$ of green synthesized Se NPs. The crystalline size of the prepared nanoparticles was determined by using Scherrer's equation as follows $D \approx 0.9 \lambda / \beta \cos \theta$, where $D$ is the crystal size, $\lambda$ is the wavelength of X-ray, $\Theta$ is the Bragg angle in radians, and $B$ is the full width at half maximum of the peak in radians.

\subsubsection{Field Emission Scanning Electron Microscopy and EDX}

The morphology of synthesized Se NPs was observed by field emission scanning electron microscopy and the elemental composition was recorded (ZEISS EVO-MA 10, Oberkochen, Germany).

\subsubsection{Transmission Electron Microscopy}

Transmission electron micrographs were recorded using a JEOL JSM 1200EX-II transmission electron microscope equipped with an electron diffraction pattern.

\subsection{Biological Application}

The advancement of the green production of nanoparticles using plant extract performs a significant role on free radical and bacterial-mediated disease. Green synthesized nanoparticles play a vital role in chemotherapy, where high doses lead toxicity to normal tissue and the immune system [18].

\subsubsection{Antioxidant Activity}

DPPH Radical Scavenging Activity The 2, 2-diphenyl-1picrylhydrazyl (DPPH) radical scavenging assay was determined by the method of [19] with some modifications. The
DPPH scavenging ability was calculated by the following formula:

DPPH RSA $(\%)$

$$
=\frac{\text { Absorbance of control-Absorbance of sample }}{\text { Absorbance of control }} \times 100
$$

\subsubsection{Antibacterial Activity}

Two Gram-positive bacterial strains (Bacillus subtilis, Staphylococcus aureus) and two Gram-negative bacterial strains (Escherichia coli, Klebsiella pneumoniae) were used. Bacterial strains were obtained from clinical laboratories in and around Salem District, Tamil Nadu. The agar well diffusion method was employed as per the modified method [20].

\subsubsection{Antiproliferative Activity}

The A549 cell line was obtained from the National Centre for Cell Sciences Repository, University of Pune, India. A549 cells were maintained in a humidified atmosphere containing $5 \% \mathrm{CO}_{2}$ at $37{ }^{\circ} \mathrm{C}$ in Dulbecco's modified Eagle's medium (DMEM) supplemented with 100 units of penicillin, $100 \mathrm{~g} / \mathrm{l}$ of streptomycin, and $10 \%$ fetal bovine serum (FBS). Briefly, A549 cell was pre-cultured in 96-well microtiter plates for $48 \mathrm{~h}$ under $5 \% \mathrm{CO}_{2}$.

The optical density (OD) value was used to calculate the percentage of cell viability by using the following formula:

Percentage of cell viability $=$ OD value of experimental NPs treated sample/OD value of experimental untreated sample 100

MTT Assay $10 \times$ stock MTT was prepared by dissolving in PBS (phosphate-buffered saline) at a concentration of $5 \mathrm{mg} / \mathrm{ml}$ and filtering through a $0.45-\mu \mathrm{m}$ filter. The growth medium of the confluent cells was removed, then $100 \mu$ of MTT was added to each well. Following incubation at $37^{\circ} \mathrm{C}$ with $5 \% \mathrm{CO}_{2}$ for $5 \mathrm{~h}, 100 \mu \mathrm{l}$ of MTT was replaced by $100 \mu \mathrm{DMSO}$ and mixed to release the color from the cells. The optical density was measured at $540 \mathrm{~nm}$ using an ELISA reader (Thermo Multiskan EX, USA) to elevate live cells [21].

\subsection{Photocatalytic Degradation of Dye}

The photocatalytic activity of green synthesized Se NPs was analyzed by degradation of methylene blue (MB) under solar irradiation [22].

\subsection{Statistical Analysis}

Statistical analyses were conducted using the SPPS software (16.0 version). Analysis of variance (ANOVA) in a completely randomized design and Tukey's multiple range tests were used to compare any significant differences between samples. Values were expressed as mean \pm standard deviation. All 
determinations were done at least in triplicate and all were averaged. The confident limits were based on $95 \%(p<0.05)$.

\section{Result and Discussion}

\subsection{Qualitative Phytochemical Analysis}

\subsubsection{Phytochemical Screening}

The qualitative phytochemical analysis of W. somnifera leaves aqueous extract is shown in Table 1. It revealed the presence of enormous active constituents like alkaloids, flavonoids, phenolics, tannins and terpenoids.

Aqueous leaves extract exhibited rich source of flavonoids and phenolics, possessing reducing functional groups. These functional groups may play role in reducing selenious acid to Se NPs. Biomolecules can serve as an active biocatalyst in producing fabricated nanomaterials. These can additionally act as natural stabilizers to nanoparticles. The plant-mediated synthesis reduces the toxic chemicals and leads to an ecofriendly synthesis of NPs [23]. W. somnifera leaves (Indian chemotype) contains withanolides, alkaloids, many free amino acids, flavonoids, glycosides, glucose, tannins, and chlorogenic acid [24]. These active phytochemicals may be combined with the metal solution to give the nanoparticles.

\subsubsection{Quantitative Phytochemical Analysis}

The biologically effective plant secondary metabolite phenolic compounds are present in the $W$. somnifera leaves aqueous extract and is estimated by using gallic acid as standard which is having the appreciable amount of phenolic content. The total phenolic content of aqueous leaves extract is in the range of $12.74 \pm$

Table 1 Qualitative phytochemical analysis of Withania somnifera leaves aqueous extract

\begin{tabular}{lll}
\hline S. no. & Phytochemical constituents & Aqueous extract \\
\hline 1 & Alkaloids & ++ \\
2 & Flavonoids & +++ \\
3 & Flavonol & - \\
4 & Phenolics & ++ \\
5 & Tannins & ++ \\
6 & Steroids & - \\
7 & Proteins & - \\
8 & Saponins & - \\
9 & Terpenoids & + \\
10 & Glycosides & - \\
\hline
\end{tabular}

+++, copiously present; ++, moderately present; +, slightly present; -, absent
Table 2 Total phenolic, flavonoids, and tannin contents of Withania somnifera leaves extract

\begin{tabular}{lllc}
\hline S. no. & $\begin{array}{l}\text { Phytochemical } \\
\text { constituents }\end{array}$ & \multicolumn{2}{l}{ Withania somnifera leaves aqueous extract } \\
\cline { 3 - 4 } & & Absorbance $(\mathrm{nm})$ & Content $(\mu \mathrm{g} / \mathrm{mg})$ \\
\hline 1 & Total phenolic & $0.234 \pm 0.004$ & $12.74 \pm 0.28$ \\
2 & Total flavonoid & $0.154 \pm 0.001$ & $40.54 \pm 0.24$ \\
3 & Total tannins & $0.041 \pm 0.016$ & $156.33 \pm 14.53$ \\
\hline
\end{tabular}

$0.28 \mu \mathrm{g} / \mathrm{ml}$ as shown in Table 2. The flavonoid content of $W$. somnifera aqueous leave extracts was observed by using catechin equivalent. The results show that the aqueous leave extract recorded the amount of flavonoid content is $40.54 \pm 0.23 \mu \mathrm{g} / \mathrm{ml}$. The tannin content is rich in the extract $(156.33 \pm 14.53 \mu \mathrm{g} / \mathrm{mg})$ and is encouraging to stabilize the metal nanoparticles due to their electron-donating capability and capping property of the tannin compounds. This plant phytochemical-mediated synthesis of metal nanoparticles may be a sign of its antioxidant potential by the deposition of bioactive compounds on their surface.

\subsection{Synthesis of Selenium Nanoparticles}

W. somnifera leaf extract is added to selenious acid solution. The color changes from colorless selenious acid to ruby red color. Synthesized Se NPs are characterized by using parameter techniques such as UV-Visible spectroscopy, FT-IR, field emission scanning electron microscopy (FE-SEM), EDX and transmission electron microscopy (TEM) and to evaluate the antioxidant, antibacterial and photocatalytic activity.

\subsection{Characterization of Selenium Nanoparticles}

\subsubsection{UV-Vis Spectra Analysis}

UV-Visible spectroscopy was used to examine the formation of Se NPs. Figure 1a shows a plodding increase in absorbance at $320 \mathrm{~nm}$ with respect to time of reaction, confirming the synthesis of Se NPs. Se NP formation was visually identified by the color change of reaction mixture from transparent into ruby red with time. After $24 \mathrm{~h}$ of incubation, no more color change was observed. This observation revealed the completely formed Se NPs by biomolecules of $W$. somnifera leaves. The UV-Visible spectroscopy analysis Fig. 1a revealed the absorption maximum $\left(\lambda_{\max }\right)$ at $320 \mathrm{~nm}$ after $24 \mathrm{~h}$ and was attributed to the surface plasmon resonance (SPR) of Se NPs. The UV-Visible absorption maximum between 200 and $400 \mathrm{~nm}$ was due to the formation of SPR of Se NPs [25].

From the optical absorption spectra, the direct optical band gap was calculated using

$$
(\alpha \mathrm{h} v)^{\mathrm{n}}=\mathrm{A}\left(\mathrm{h} v-E_{\mathrm{g}}\right)
$$



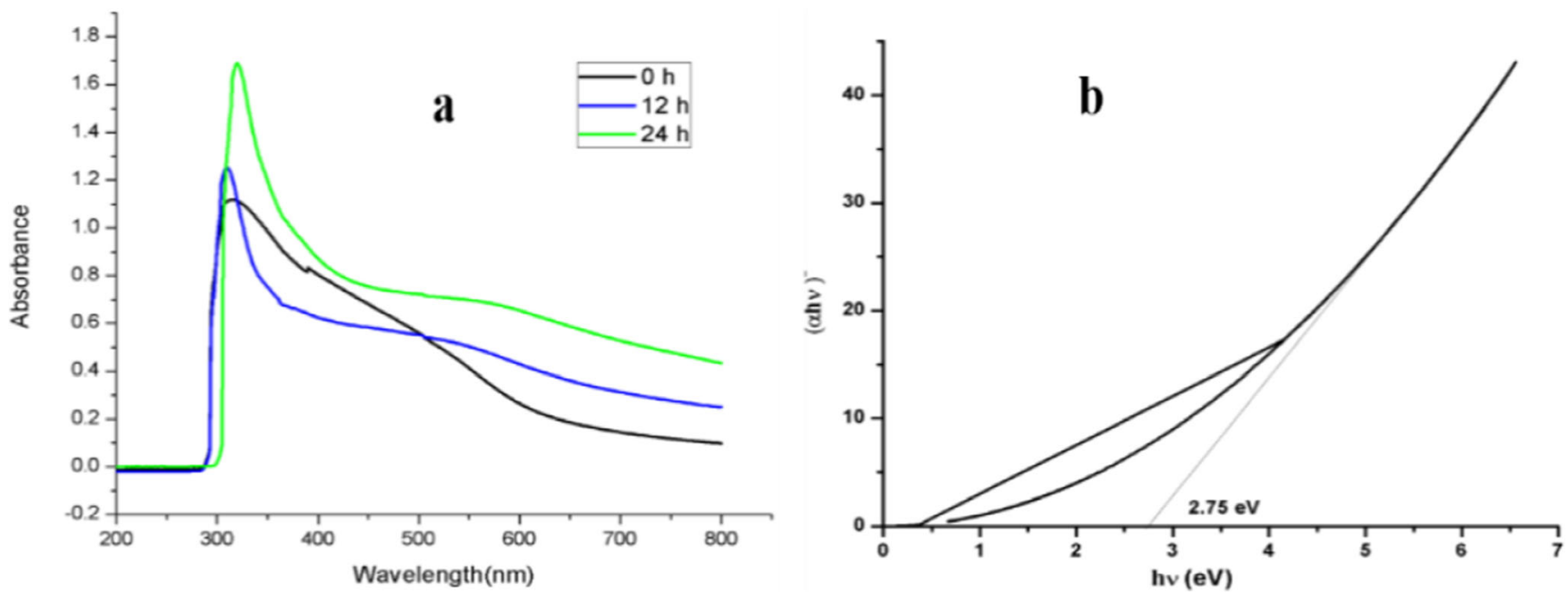

Fig. 1 a UV-Visible spectrum at different time intervals (0, 12, and $24 \mathrm{~h})$. b Band gap energy of selenium NPs at $24 \mathrm{~h}$

where $E_{\mathrm{g}}$ is the energy gap, $\alpha$ is the absorption coefficient, $\mathrm{h}$ is Planck's constant, $v$ is the frequency of light, $A$ is the constant of proportionality, and $n=2$ for direct band gap energy [26]. From the plots (Fig. 1b), band gap energy is found to be $2.75 \mathrm{eV}$ for as-prepared $24-\mathrm{h}$ aging time respectively. It was observed that the band gap energy of green synthesized Se NPs is higher than that of bulk $\alpha$-Se $(2.0 \mathrm{eV})$ and commercial Se powder
$(1.8 \mathrm{eV})$ [27] due to quantum size effect, similar to the early reports of Se NPs band gap $2.72 \mathrm{eV}$ at 24-h aging time. Green synthesis of Se nanoparticles band gap energy $(2.75 \mathrm{eV})$ is large than that of commercial Se nanoparticles $(1.8 \mathrm{eV})$ whereas green $\mathrm{Se}$ nanoparticles interact with plant biomolecules so less toxic, low cost effect and very safe to the environment. But commercial Se nanoparticles are synthesized by various chemical

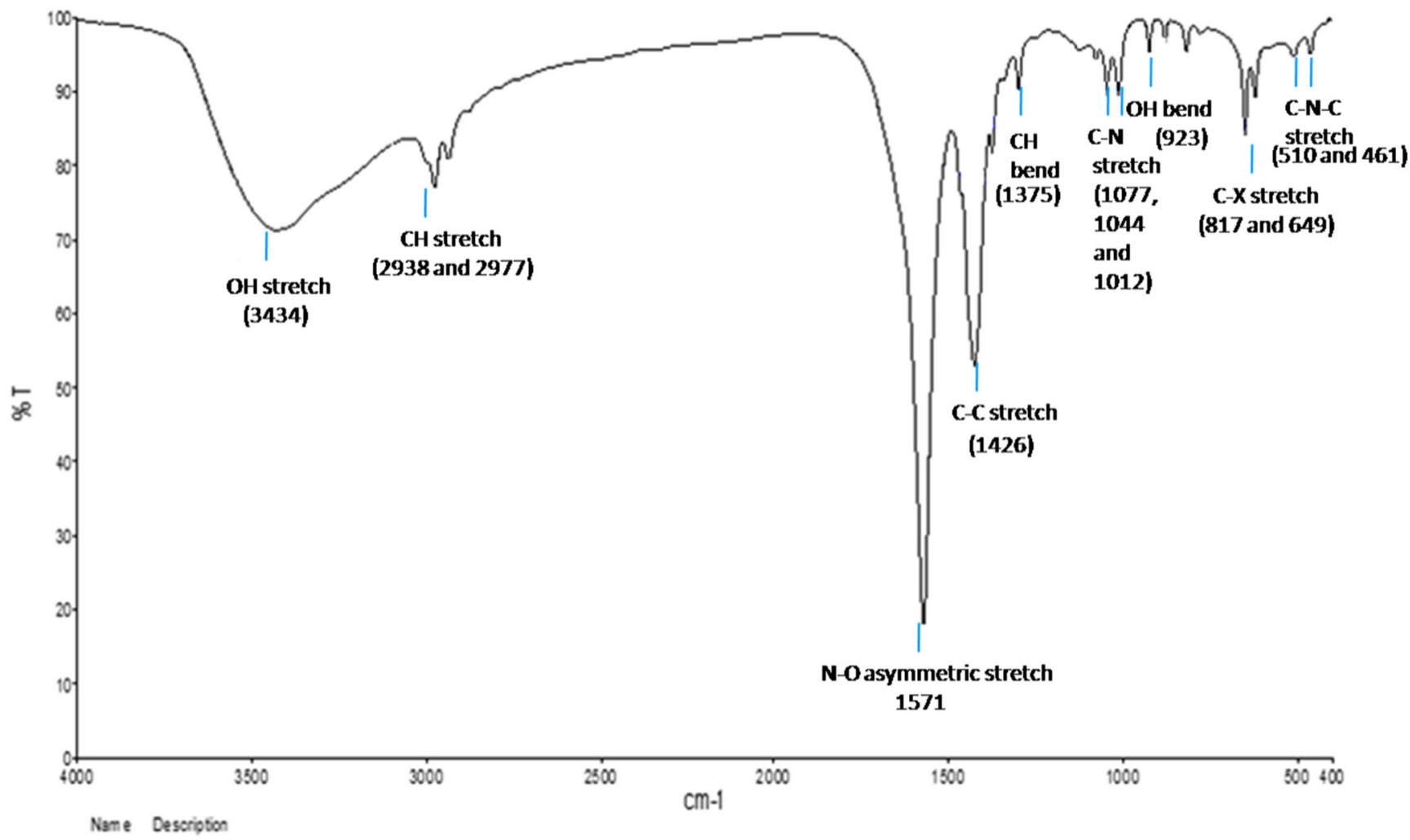

Fig. 2 FT-IR spectra of synthesized selenium NPs 
Fig. 3 XRD spectra of synthesized selenium NPs

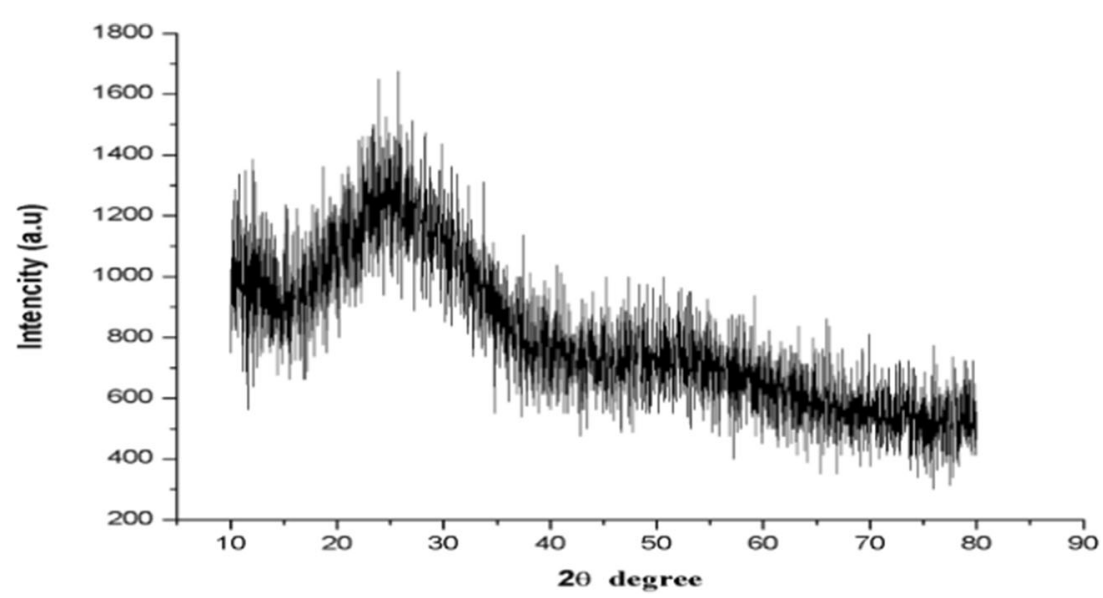

methods. Previous reports also demonstrate that green Se nanoparticles are better for the environment [28].

\subsubsection{FT-IR Analysis}

The result of FT-IR analysis of synthesized Se NPs is depicted in Fig. 2. A broad peak observed at $3434 \mathrm{~cm}^{-1}$ corresponds to $\mathrm{O}-\mathrm{H}$ stretch alcohols and phenols.
Absorption peak at 2938 and $2977 \mathrm{~cm}^{-1}$ corresponds to $\mathrm{C}-\mathrm{H}$ stretch alkynes. The band at $1571 \mathrm{~cm}^{-1}$ is due to $\mathrm{N}-$ $\mathrm{O}$ asymmetric stretch nitro compounds. The strong band at $1426 \mathrm{~cm}^{-1}$ is due to $\mathrm{C}-\mathrm{C}$ stretching (in ring) of aromatics. The sharp peak at $1375 \mathrm{~cm}^{-1}$ is attributed to the $\mathrm{C}-$ $\mathrm{H}$ bending in alkanes and the 1077, 1044, and $1012 \mathrm{~cm}^{-1}$ corresponds to the $\mathrm{C}-\mathrm{N}$ stretching of the amines. The band at $923 \mathrm{~cm}^{-1}$ is due to the $\mathrm{O}-\mathrm{H}$ bend of carboxylic
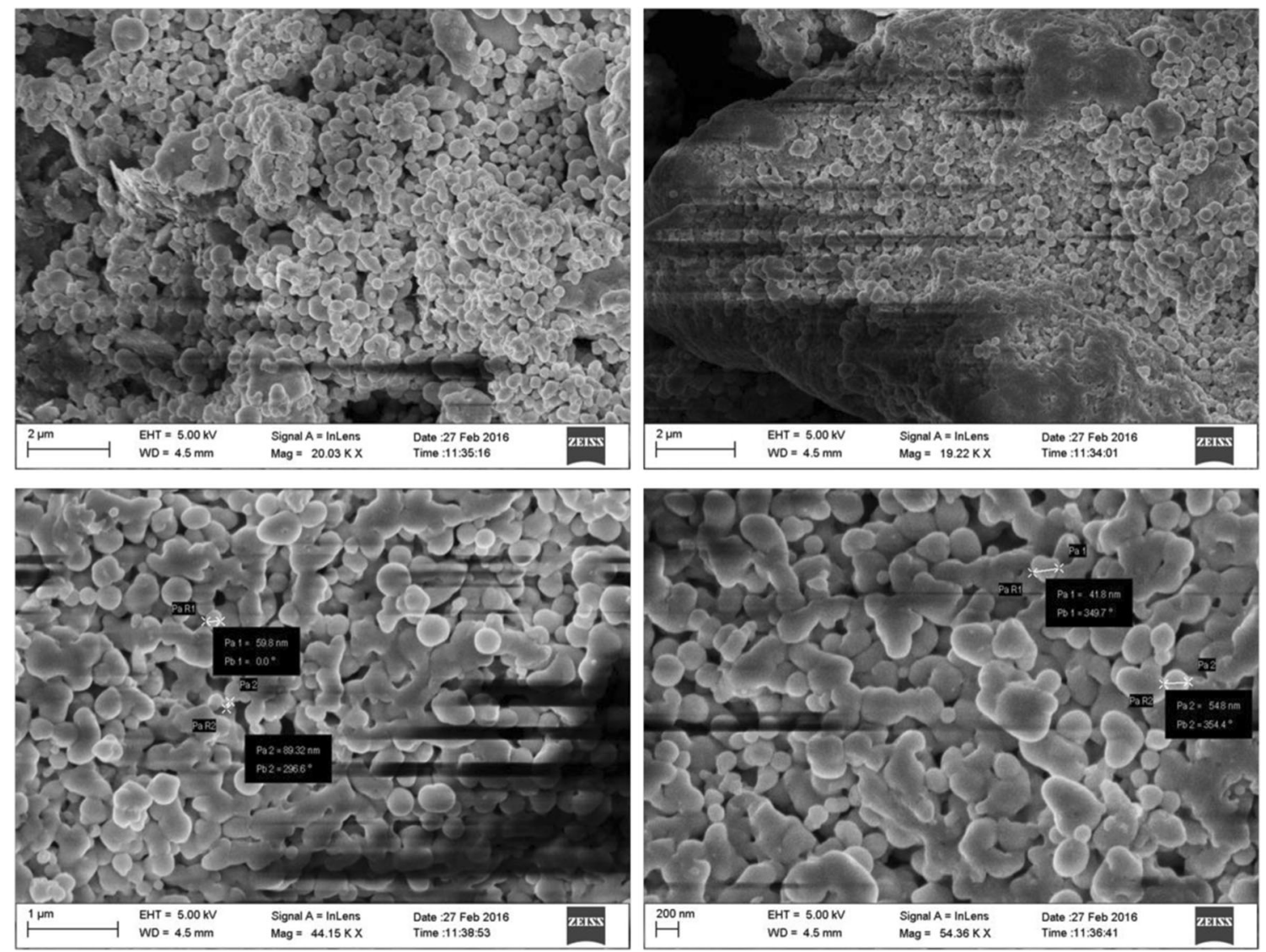

Fig. 4 FE-SEM images of selenium NPs 


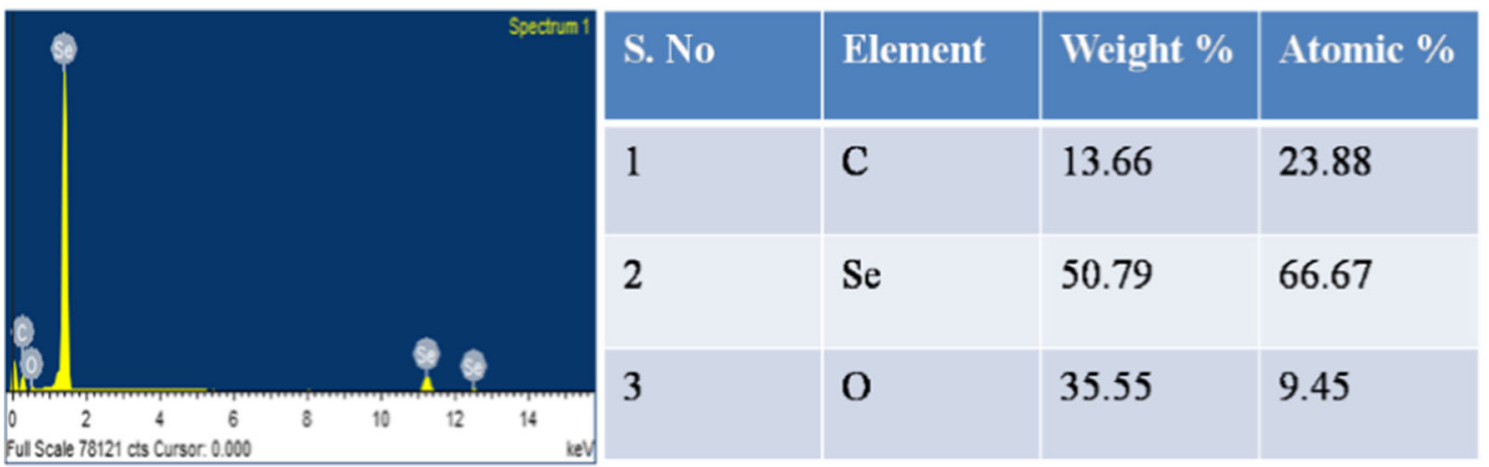

Fig. 5 EDX spectra of selenium NPs

acids. $\mathrm{C}-\mathrm{X}$ stretching in alkyl halides causes a band at 817 and $649 \mathrm{~cm}^{-1}$ and the weak band at 510 and $461 \mathrm{~cm}^{-1}$ is the result of $\mathrm{C}-\mathrm{N}-\mathrm{C}$ bending in amines.

This result indicates the presence of various functional groups as biomolecules which may be responsible for both reduction and stabilization of the Se NPs. Previous reports have also suggested the role of phytochemicals as a stabilizing agent for the synthesis of metal NPs [29]. However, the identity of the active molecules responsible for the synthesis of the Se NPs needs further validation.

\subsubsection{XRD Analysis}

X-ray diffraction pattern result reveals that Se NPs are amorphous in nature (Fig. 3). The result matches with the previous reported values [30]. Nonetheless, the X-ray diffraction pattern could be indexed according to the trigonal phase of selenium, which could be realized from the appearance of the diffraction peaks characteristic of these mentioned phases of Se having lattice constants $a=4.366 \mathrm{~A}^{\circ}$ and $c=4.956 \mathrm{~A}^{\circ}$ (JCPDS file no. 06-362) [31, 32]. The prepared Se nanoparticle-calculated crystalline size is $58 \mathrm{~nm}$ using Scherrer's equation.

\subsubsection{Field Emission Scanning Electron Microscopic Analysis}

Scanning electron microscopy is employed to analyze the shape of the Se NPs synthesized by green method using $W$. somnifera leaves aqueous extract. Figure 4 shows FE-SEM image of Se NP greater numbers of the agglomerated spherical particles within the diameter range 45-90 nm. These particles were well distributed with aggregation. Therefore, it has been suggested that nanoparticle aggregation is dominant over the process of reduction and primary nucleation of reduced atoms. This may perhaps be related to the fact that a larger number of functional groups ( $W$. somnifera leaves aqueous extract) bind and nucleate selenious acid ions. The most accessible metal ions are apparently involved in a smaller number of nucleation events, which leads to agglomeration of the metal [33]. Previous report showed agglomerate NPs exhibit higher biological activity $[34,35]$. The synthesized $W$. somnifera leaves aqueous extract-mediated Se NPs may have efficient applications in pharmacology.

\subsubsection{Energy-Dispersive X-ray Spectroscopy Analysis}

EDX was employed to analyze the elemental chemical composition of Se NPs Fig. 5. Three signals could be observed
Fig. 6 TEM image and selected area electron diffraction patterns of selenium NPs

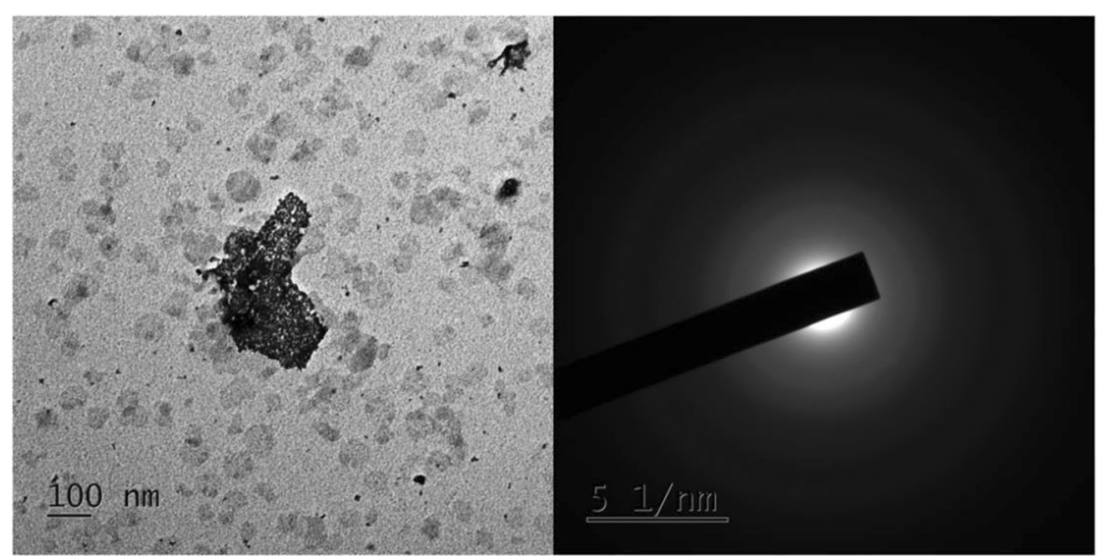


from the energy-dispersive X-ray analysis: a strong signal from the Se atom (50.79\%) together with $\mathrm{O}$ atom (35.55\%) and $\mathrm{C}$ atom $13.66 \%$. There were no evident peaks for other elements or impurities.

\subsubsection{Transmission Electron Microscopy}

The synthesized Se NPs obtained agglomerated particles as inferred by transmission electron microscopy with selected area electron diffraction patterns (amorphous nature) (Fig. 6) [36].

\subsection{Biological Application}

\subsubsection{Antioxidant Activity of Selenium Nanoparticles}

Evaluation of antioxidant activities by DPPH radical is a stable free radical [37]. The antioxidant activity of formulated Se NPs was estimated by comparing the percentage inhibition of DPPH radicals and ascorbic acid. The DPPH radical scavenging activity of Se NPs increased with increase in concentration. DPPH free radical scavenging assays are shown in Fig. 6. Both Se NPs and plant extract showed the activity to scavenge DPPH free radicals in a dose-dependent manner at 20 and $100 \mathrm{mg} / \mathrm{ml}$. Se NPs showed moderate antioxidant activity when compared with plant extract and ascorbic acid. These results revealed that the Se NPs act as an efficient radical scavenger.

In vivo free radicals are the products of normal cellular metabolism and serve as signaling and regulatory molecules. However, excess free radicals will result in the damage of cellular compounds and finally inhibit their normal function. To date, some studies have suggested that the amorphous Se NP have excellent antioxidant activity [38].

\subsubsection{Antibacterial Activity}

Infectious diseases are major causes of morbidity and mortality worldwide. At the present time, several pathogenic microorganisms have developed resistance to currently
Table 4 A549 cell line $\mathrm{IC}_{50}$ values

\begin{tabular}{lc}
\hline Concentration $(\mu \mathrm{g} / \mathrm{ml})$ & $\begin{array}{c}\text { \% cell viability } \\
\text { A549 cell line }\end{array}$ \\
\hline Control & 100 \\
12.5 & 78.2 \\
25 & 49.2 \\
50 & 38.4 \\
75 & 21.7 \\
100 & 14.8 \\
$\mathrm{IC}_{50}$ & 49.2 \\
\hline
\end{tabular}

available commercial antibiotics and also cause adverse impact on health [39]. Hence, there is an urgent need to discover a new, more active, and safe antimicrobial agent. Recent attention has been paid to the use of nanoparticles to control diseases due to the antimicrobial properties of these nanoparticles.

The antibacterial activity of Se NPs was carried out on four pathogenic bacterial strains, Bacillus subtilis, Escherichia coli, Klebsiella pneumoniae and Staphylococcus aureus. An antibacterial drug, ciprofloxacin, was used as positive control in Table 3. Se NPs exhibited considerable activity against Klebsiella pneumoniae and Bacillus subtilis with zone of inhibition 14 and $12 \mathrm{~mm}$ but no activity against Escherichia coli pathogen. The higher zone of inhibition is observed in Staphylococcus aureus $(19.66 \mathrm{~mm})$ by Se NPs which may (efficiency to Staphylococcus aureus pathogen) serve as an antibacterial agent. The use of green synthesis of metal nanoparticles is going to be of significant importance in medicinal field.

\subsubsection{Antiproliferative Activity}

Active targeting of nanoparticles, a strategy of cancer nanotechnology, is usually achieved by combination of a targeting component to the surface of nanoparticles which leads to the preferential accumulation of nanoparticles in tumor sites, individual cancer cells, or intracellular organelles inside cancer cells [40].

Table 3 Antibacterial activity of selenium NPs

\begin{tabular}{llllll}
\hline S. No & Bacteria & Organisms & \multicolumn{2}{l}{ Diameter of zone of inhibition (in mm) } \\
\cline { 3 - 5 } & & WS- Se NPs & Positive control $^{\mathrm{a}}$ & Negative control $^{\mathrm{b}}$ \\
\hline 1 & \multirow{2}{*}{ Gram-positive bacteria } & B. subtilis & $14.00 \pm 0.00$ & $27.25 \pm 2.62$ & $00.00 \pm 0.00$ \\
2 & & S. aureus & $19.66 \pm 0.57$ & $34.25 \pm 1.70$ & $00.00 \pm 0.00$ \\
3 & Gram-negative bacteria & E. coli & $00.00 \pm 0.00$ & $00.00 \pm 0.00$ & $00.00 \pm 0.00$ \\
4 & & K. pneumoniae & $12.00 \pm 0.00$ & $28.75 \pm 1.25$ & $00.00 \pm 0.00$ \\
\hline
\end{tabular}

The values are mean of triplicates with $( \pm$ ) standard deviation (mean $\pm \mathrm{SD} ; n=3$ )

${ }^{\text {a }}$ Ciprofloxacin $(1 \mathrm{mg} / \mathrm{ml})$

${ }^{\mathrm{b}} \mathrm{DMSO}(50 \mu \mathrm{l} / \mathrm{well})$ 
Fig. 7 Antiproliferative activity of selenium nanoparticles by MTT assay

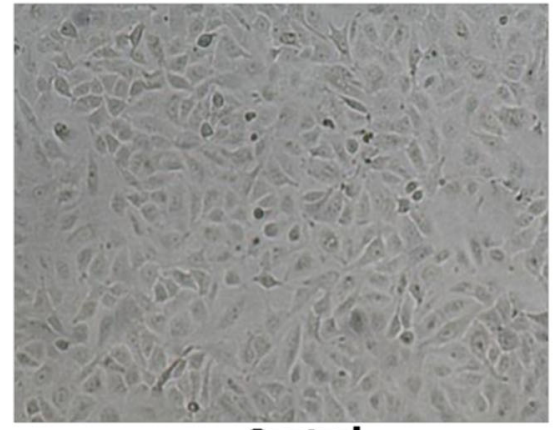

\section{Control}

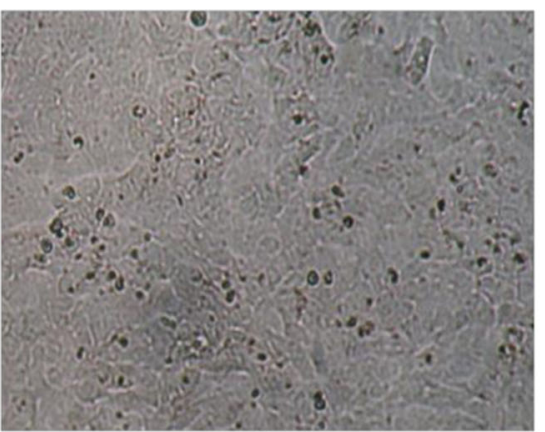

$25 \mu \mathrm{g}$

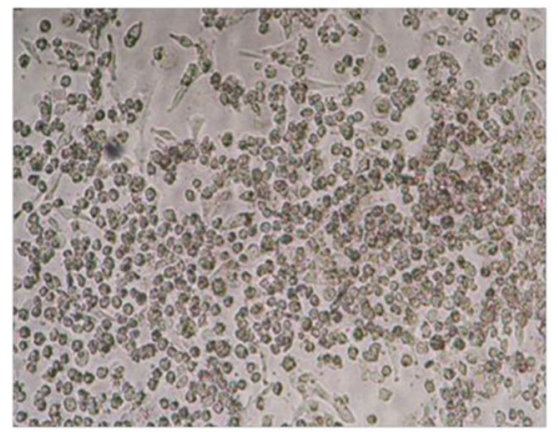

$75 \mu \mathrm{g}$

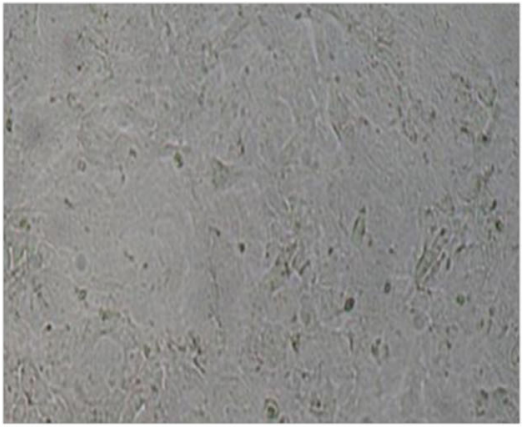

$10 \mu \mathrm{g}$

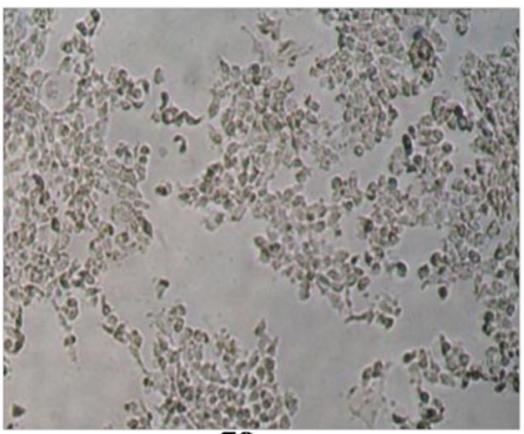

$50 \mu \mathrm{g}$

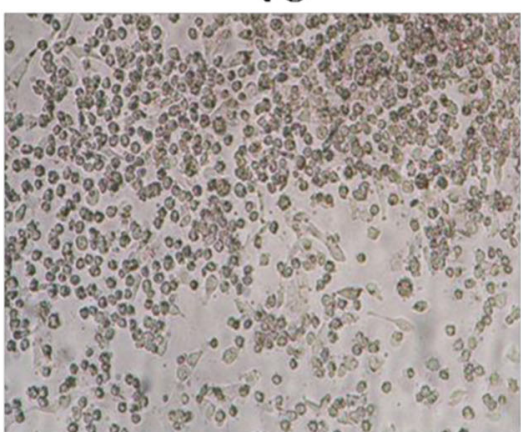

$100 \mu g$
Green synthesized Se NPs were treated on adenocarcinomic human alveolar basal epithelial cancer cell (A549) by MTT assay. The electron micrographs revealed a uniform intracellular distribution of Se NPs both in cancer cells. Se NP-treated cells exhibited chromosome instability and mitotic arrest in human alveolar basal
Fig. 8 DPPH radical scavenging activity of selenium NPs

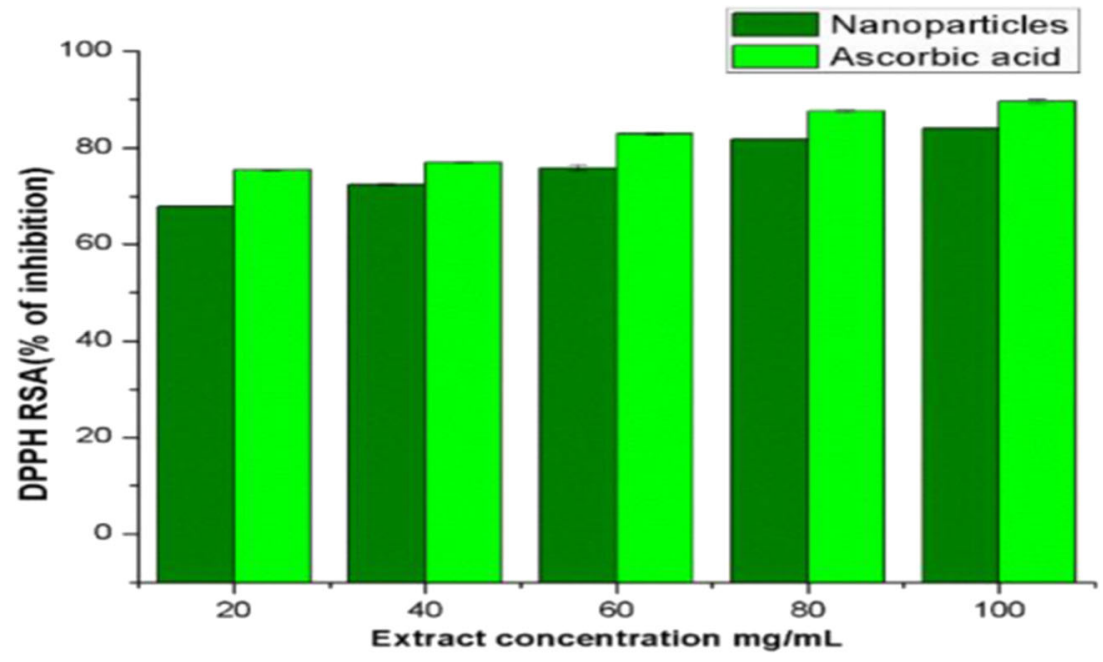


Fig. 9 Absorbance spectra of MB in the presence of selenium NPs

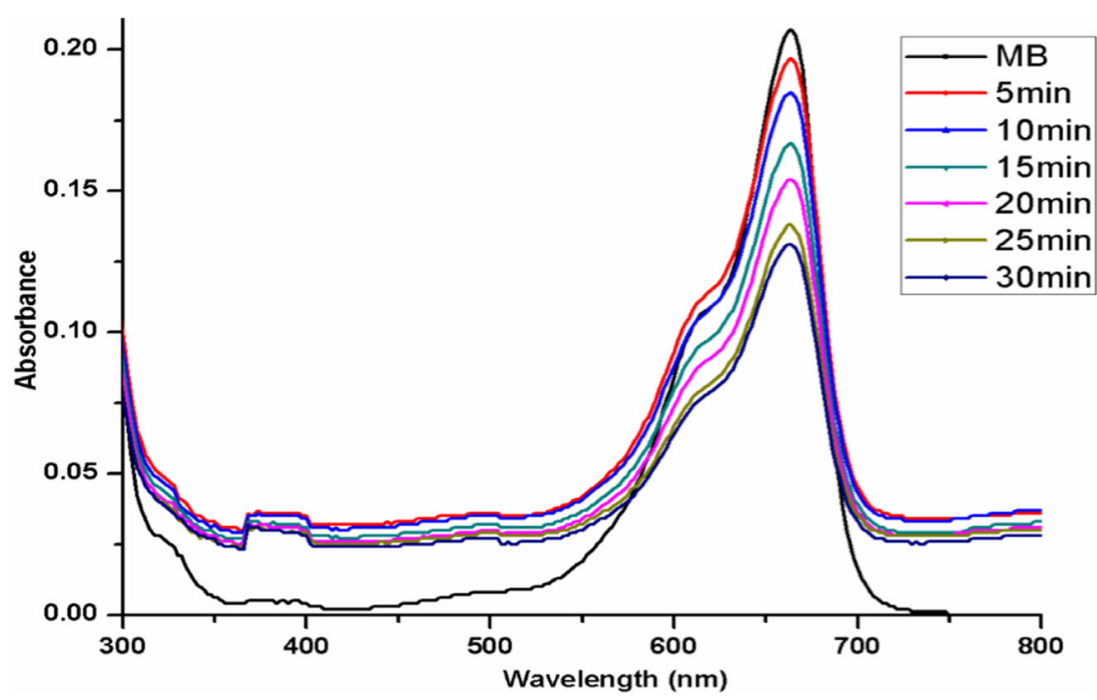

epithelial cancer cells. The antiproliferative activity of Se NPs significantly inhibited the growth of A549 cells and was observed on treatment with Se NPs, as shown in Fig. 4. The half maximum inhibitory concentration $\left(\mathrm{IC}_{50}\right)$ was observed at $25 \mu \mathrm{g}$. The cellular morphological changes of A549 cells exposed to MA-Se NPs for $72 \mathrm{~h}$ were also examined by a phase-contrast microscopy, showing dosedependent cell number decrease and loss of intercellular contact, while control group retained its number and shape (Table 4).

\subsubsection{Photocatalytic Activity}

Photocatalytic activity of the synthesized Se NPs was evaluated by dye degradation of MB under sunlight. Degradation of dye was visually identified by gradual change in the color from deep blue to colorless dye solution. The characteristic absorption peak was found at $657 \mathrm{~nm}$ for pure methylene blue dye solution. The dye degradation in presence of synthesized Se NPs was verified by the decrease of the peak intensity at $657 \mathrm{~nm}$ during 30-min exposure in solar light shown in Fig. 7.

In the process of photocatalytic oxidation degradation of dye, the main active species are holes, superoxide radicals, and hydroxyl radical [41]. The photocatalytic study concluded that these Se NPs have efficiency to degrade MB under solar irradiation (Figs. 8 and 9). Therefore, they can find application in water treatment plants and textile industries.

\section{Conclusion}

Withania somnifera leaves extract possesses active constituents like alkaloids, flavonoids, phenolics, tannins, and terpenoids and acts as good reducing agents for the preparation of Se NPs. FT-IR (this analysis will help to study the functional groups present in the samples) result shows various functional groups present in the prepared Se nanoparticles because numerous phytochemicals interact with nanoparticles. Nanoparticles in aqueous (colloidal) solutions are not thermodynamically stable and hence capping of the phytoconstituents provides stability to Se NPs as evident from FT-IR studies and XRD exhibited amorphous nature of Se NPs. Selenium have high purity and are crystalline in nature, identified using EDX. The DPPH scavenging analysis of the spherical amorphous Se NPs has significant antioxidant activity and serves as a potential antibacterial agent to treat diseases caused by bacteria. Se NPs have antiproliferative activity against A549 lung carcinoma cells with $50 \%$ inhibitory concentration value $\left(\mathrm{IC}_{50}\right)$ at $25 \mu \mathrm{g} / \mathrm{ml}$. These results suggest that Se NPs possess great growth control against cancer cells which indicated their potential in medical applications. Moreover, the photocatalytic study concludes that these green Se NPs have efficiently degraded MB under sunlight irradiation. Therefore, they can find application in water treatment plants and textile industries. Hence, the combination of phytocompounds and nanoparticles serves an efficient role in its multifaceted pharmacological properties.

Funding Information Funding was provided by the Adi Dravidar Welfare Scholarship (Govt. of Tamil Nadu).

\section{Compliance with Ethical Standards}

Conflict of Interest The authors declare that they have no conflicts of interest.

Open Access This article is distributed under the terms of the Creative Commons Attribution 4.0 International License (http:// creativecommons.org/licenses/by/4.0/), which permits unrestricted use, 
distribution, and reproduction in any medium, provided you give appropriate credit to the original author(s) and the source, provide a link to the Creative Commons license, and indicate if changes were made.

\section{References}

1. Fazal, S., Jayasree, A., Sasidharan, S., Koyakutty, M., Nair, S. V., \& Menon, D. (2014). Green synthesis of anisotropic gold nanoparticles for photothermal therapy of cancer. ACS Applied Materials \& Interfaces, 6(11), 8080-8089. https://doi.org/10.1021/am500302t.

2. Genevieve, A., Kahrilas Laura, M., Wally, S. J., Fredrick Michael, H., Amy, L., Prieto, J. E., \& Owens. (2014). Microwave-assisted green synthesis of silver nanoparticles using orange peel extract. ACS Sustainable Chemistry \& Engineering, 2(3), 367-376. https://doi.org/10.1021/sc4003664.

3. Ramamurthy, C. H., Sampath, K. S., Arunkumar, P., Suresh kumar, M., Sujatha, V., Premkumar, K., \& Thirunavukkarasu, C. (2013). Green synthesis and characterization of selenium nanoparticles and its augmented cytotoxicity with doxorubicin on cancer cells. Bioprocess and Biosystems Engineering, 36(8), 1131-1139. https://doi.org/10.1007/s00449-012-0867-1.

4. Umaralikhan, L., \& Jamal mohamed jaffar, M. (2016). Green synthesis of Mgo nanoparticles and it antibacterial activity. Iranian Journal of Science and Technology Transactions a Science, 12, 1-9. https://doi.org/10.1007/s40995-016-0041-8.

5. Fardood, S. T., \& Ramazani, A. (2016). Green synthesis and characterization of copper oxide nanoparticles using coffee powder extract. Journal Nanostructures, 6(2), 167-171. https://doi.org/10. 7508/jns.2016.02.009.

6. Suresh, D., Nethravathi, P. C., Udayabhanu, H., Rajanaika, H., Nagabhushana, H., \& Sharma, S. C. (2015). Green synthesis of multifunctional zinc oxide nanoparticles using Cassia fistula plant extract and their photodegradative, antioxidant and antibacterial activities. Materials Science in Semiconductor Processing, 31, 446-454. https://doi.org/10.1016/j.mssp.2014.12.023.

7. Monalisha, R., Swati, S., Panda, N. K., \& Dhal. (2014). Synthesis of silver nanoparticles from plant extract and its application in cancer treatment: a review. International Journal of Plant Animal and Environmental Sciences, 4(3), 137-145.

8. Verma, S., Hoffmann, F. W., Kumar, M., Huang, Z., Roe, K., Nguyen-Wu, E., Hashimoto, A. S., \& Hoffmann, P. R. (2011). Selenoprotein $\mathrm{K}$ knockout mice exhibit deficient calcium flux in immune cells and impaired immune responses. Journal of Immunology, 186(4), 2127-2137. https://doi.org/10.4049/ jimmunol.1002878.

9. Li, Y., Li, X., Zheng, W., Fan, C., Zhang, Y., \& Chen, T. (2013). Functionalized selenium nanoparticles with nephroprotective activity, the important roles of ROS-mediated signaling pathways. Journal of Materials Chemistry B, 1, 6365-6372. https://doi.org/ 10.1039/C3TB21168A.

10. Ghada, M., Khiralla, B. A., \& El-Deeb, A. B. (2015). Antimicrobial and antibiofilm effects of selenium nanoparticles on some foodborne pathogens. Journal of Food Science and Technology, 63(2), 1001-1007. https://doi.org/10.1016/j.lwt.2015.03.086.

11. Bashir, H. S., Mohammed, A. M., Magsoud, A. S., \& Shaoub, A. M. (2013). Isolation of three flavonoids from Withania somnifera leaves (Solanaceae) and their antimicrobial activities. Journal of Forest Products \& Industries, 2(5), 39-45.

12. Supe, U., Dhote, F., \& Roymon, M. G. (2011). A review on micro propagation of Withania somnifera - a medicinal plant. Journal of Agriculture, Science and Technology, 7(6), 1475-1483.
13. Umadevi, M., Rajeswari, R., sharmilarahale, C., Selvavenkadesh, S., Pushpa, R., Sampath kumar, K. P., \& Bhowmik, D. (2012). Traditional and medicinal uses of Withania somnifera. The Pharma Innovation Journal, 1(9), 106-110.

14. Onwukeame, D. N., Ikuegbvweha, T. B., \& Asonye, C. C. (2007). Evaluation of phytochemical constituents, antibacterial activities and effect of exudate of Pycanthus angolensis weld warb (myristicaceae) on corneal ulcers in rabbits. Tropical Journal of Pharmaceutical Research, 6, 725-730.

15. Barreira, J., Ferreira, I. C. F. R., Oliveira, M., \& Pereira, J. A. (2008). Antioxidant activities of the extracts from chestnut flower, leaf, skins and fruit. Food Chemistry, 107(3), 1106-1113. https:// doi.org/10.1016/j.foodchem.2007.09.030.

16. Kathirvel, A., \& Sujatha, V. (2012). Phytochemical studies, antioxidant activities and identification of active compounds using GCMS of Dryopteris cochleata leaves. Arabian Journal of Chemistry. https://doi.org/10.1016/j.arabjc.2012.03.018.

17. Oyaizu, M. (1986). Studies on products on browning reaction prepared from glucose amine. Japanese Journal of Nutrition, 44, $307-$ 315.

18. Lee, Y. K., Choi, J., Wang, W., Lee, S., Nam, T. H., Choi, W. S., Kim, C. J., Lee, J. K., Kim, S. H., Kang, S. S., \& Khang, D. (2013). Nullifying tumor efflux by prolonged endolysosome vesicles: development of low dose anticancer-carbon nanotube drug. ACS Nano, 7(10), 8484-8497. https://doi.org/10.1021/nm4041206.

19. Nagajyothi, P. C., Cha, S. J., Yang, I. J., Sreekanth, T. V., Kim, K. J., \& Shin, H. M. (2015). Antioxidant and anti-inflammatory activities of zinc oxide nanoparticles synthesized using Polygala tenuifolia root extract. Journal of Photochemistry and Photobiology B: Biology, 146, 10-17. https://doi.org/10.1016/j.jphotobiol.2015.02. 008 .

20. Srinivasan, R., Natarajan, D., \& Shivakumar, M. S. (2014). Antimicrobial and GC-MS analysis of Memecylon edule leaf extracts. International Journal of Current Pharmaceutical Review and Research, 5, 1-13.

21. Udayabhanua, N. G., Nagabhushanab, H., Basavarajb, R. B., Raghua, G. K., Sureshc, D., Rajanaikad, H., \& Sharmae, S. C. (2016). Green, non-chemical route for the synthesis of $\mathrm{ZnO}$ superstructures, evaluation of its applications towards photocatalysis, photoluminescence and bio-sensing. Crystal Growth \& Design, 16(12), 6828-6840. https://doi.org/10.1021/acs.cgd.6b00936.

22. Selvakumaran, M., Pisarcik, D. A., Bao, R., Yeung, A. T., \& Hamilton, T. C. (2003). Enhanced cisplatin cytotoxicity by disturbing the nucleotide excision repair pathway in ovarian cancer cell lines. Cancer Research, 63(6), 1311-1316.

23. Pearce, C. L., Coker, V. S., Charnock, J. M., Pattrick, R. A. D., Mosselmans, J. F. W., Law, N., Beveridge, T. J., \& Lloyd, J. R. (2008). Microbial manufacture of chalcogenide-based nanoparticles via the reduction of selenite using Veillonella atypica: an in situ exafs study. Nanotechnology, 19(15), 155603. https://doi.org/ 10.1088/0967-4484/19/15/155603.

24. Uddin, Q., Samiulla, L., Singh, V. K., \& Jamil, S. S. (2012). Phytochemical and pharmacological profile of Withania somnifera Dunal: a review. Journal of Applied Pharmaceutical Science, 2(1), 170-175.

25. Ramamurthya, C. H., Padma, M., Daisy mariyasamadanam, I., Mareeswaran, R., Suyavaran, A., Suresh kumar, M., Premkumar, K., \& Thirunavukkarasu, C. (2013). The extra cellular synthesis of gold and silver nanoparticles and their free radical scavenging and antibacterial properties. Colloids and Surfaces, B: Biointerfaces, 102, 808-815. https://doi.org/10.1016/j.colsurfb.2012.09.025.

26. Mehta, S. K., Chaudhary, S., Kumar, S., Bhasin, K. K., Torigoe, K., Sakai, H., \& Abe, M. (2008). Surfactant assisted synthesis and spectroscopic characterization of selenium nanoparticles in ambient conditions. Nanotechnology, 19(29), 295601. https://doi.org/10. 1088/0957-4484/19/29/295601. 
27. Bhatnagar, A. K., Reddy, K. V., \& Srivastava, V. (1985). Optical energy gap of amorphous selenium: effect of annealing. Journal of Physics D: Applied Physics, 18(9), L149. https://oi.org/10.1088/ 0022-3727/18/9/001.

28. Kannan, S., Mohanraj, K., Prabhu, K., Barathan, S., \& Sivakumar, G. (2014). Synthesis of selenium nanorods with assistance of biomolecule. Bulletin of Materials Science, 37(7), 1631-1635.

29. Coccia, F., Tonucci, L., Bosco, D., Bressand, M., \& d'Alessandro, N. (2012). One pot synthesis of lignin-stabilized platinum and palladium nanoparticles and their catalytic behavior on oxidation and reduction reactions. Green Chemistry, 14, 1073-1078. https://doi. org $/ 10.1039 / \mathrm{c} 2 \mathrm{gc} 16524 \mathrm{~d}$.

30. Chen, Z., Shen, Y., Xie, A., Zhu, J., Wu, Z., \& Huang, F. (2009). LCysteine-assisted controlled synthesis of selenium nanospheres and nanorods. Crystal Growth \& Design, 9(3), 1327-1333. https://doi. org $/ 10.1021 / \mathrm{cg} 800398 \mathrm{~b}$.

31. Yang, F., Tang, Q., Zhong, X., Bai, Y., Chen, T., Zhang, Y., Li, Y., \& Zheng, W. (2012). Surface decoration by Spirulina polysaccharide enhances the cellular uptake and anticancer efficacy of selenium nanoparticles. International Journal of Nanomedicine, 7, 835-844.

32. Shah, C. P., Singh, K. K., Kumar, M., \& Bajaj, P. N. (2010). Vinyl monomers-induced synthesis of polyvinyl alcohol-stabilized selenium nanoparticles. Materials Research Bulletin, 45, 56-62.

33. Sathishkumar, M., Sneha, K., \& Yun, Y. S. (2010). Immobilization of silver nanoparticles synthesized using Curcuma longa tuber powder and extract on cotton cloth for bactericidal activity. Bioresource Technology, 101(20), 7958-7965.

34. Zook, J. M., Maccuspie, R. I., Locascio, L. E., Halter, M. D., \& Elliott, J. (2011). Stable nanoparticle aggregates/agglomerates of different sizes and the effect of their size on hemolytic cytotoxicity. Nanotoxicology, 5(4), 517-530.
35. Bantz, C., Koshkina, O., Lang, T., Galla, H. J., Kirkpatrick, C. J., Stauber, R. H., \& Maskos, M. (2014). The surface properties of nanoparticles determine the agglomeration state and the size of the particles under physiological conditions. Beilstein Journal of Nanotechnology, 5, 1774-1786.

36. Kora, A. J. (2018). Bacillus cereus, selenite-reducing bacterium from contaminated lake of an industrial area: a renewable nanofactory for the synthesis of selenium nanoparticles. Bioresources Bioprocessing, 5(30), 1-12.

37. Molyneux, P. (2004). The use of the stable free radical di phenyl picryl hydrazyl (DPPH) for estimating antioxidant activity. Songklanakarin Journal of Science and Technology, 26(2), 211219.

38. Li, Y., Li, X., Wong, Y. S., Chen, T., Zhang, H., Liu, C., \& Zheng, W. (2011). The reversal of cisplatin-induced nephrotoxicity by selenium nanoparticles functionalized with 11-mercapto-1undecanol by inhibition of ROS-mediated apoptosis. Biomaterials, 32(34), 9068-9076. https://doi.org/10.1016/j. biomaterials.2011.08.001.

39. Lee ventola, C. (2015). The antibiotic resistance crisis part 1: causes and threats. PT, 4(40), 277-283.

40. Sinha, R., Kim, G. J., Nie, S., \& Shin, D. M. (2006). Nanotechnology in cancer therapeutics: bioconjugated nanoparticles for drug delivery. Molecular Cancer Therapeutics, 5(8), 1909-1917. https://doi.org/10.1158/1535-7163.MCT-06-0141.

41. Zhang, Y., Zhou, J., Li, Z., \& Feng, Q. (2017). Photodegradation pathway of rhodamine B with novel Au nanorods @ ZnO microspheres driven by visible light irradiation. Journal of Materials Science, 53, 3149-3162. https://doi.org/10.1007/s10853-0171779-x. 